\title{
Differences of Salivary Superoxide Dismutase Levels in Gingivitis and Periodontitis Patients: Periodontal Installation, Faculty Of Dentistry, Universitas Sumatera Utara
}

\author{
Armia Syahputra*, Krisnamurthy, Chrisnawati Sinaga \\ Department of Periodontics, Universitas Sumatera Utara, Medan, Indonesia \\ *Email: mios_drg_01@yahoo.co.id
}

\begin{abstract}
Periodontal disease can be divided into gingivitis and periodontitis. In oral tissue, polymorphonuclear leukocytes (PMNs) are the first defense against pathogenic microorganisms. Activated PMNs produce large amounts of reactive oxygen species (ROS) that cause the destruction of periodontal tissue via various mechanisms and oxidative stress. Hosts are able to release antioxidants to eliminate ROS; one of the most effective antioxidants is superoxide dismutase (SOD). Previous studies have suggested that antioxidant levels, including SOD, are higher for periodontitis than for gingivitis or healthy people. In contrast, previous research has also found that levels of antioxidants, including SOD, were higher in healthy people followed by gingivitis and then periodontitis. Objectives: The purpose of this study was to determine differences in salivary SOD levels in patients with gingivitis and periodontitis. This was an analytic study with a cross-sectional design. There were 44 subjects: 22 with gingivitis and 22 with periodontitis recruited from the periodontal installation of the Dentistry Faculty, University of Sumatera Utara, Medan, Indonesia. All subjects' periodontal tissues were examined to determine their periodontal status. Subjects were sampled by collecting $2 \mathrm{ml}$ of saliva and salivary SOD levels were measured using a spectrophotometer. The data was analysed using an independent t-test. Result: The results showed a significant difference $(\mathrm{p}<0.05)$ between salivary SOD levels of gingivitis patients $(12.44 \pm 4.43, \mathrm{p}=0.377)$ and periodontitis patients $(9.19 \pm 3.74, \mathrm{p}=0.228)$. Salivary SOD levels were higher in patients with gingivitis than for patients with periodontitis.
\end{abstract}

Keywords: saliva, superoxide dismutase, antioxidant, gingivitis, periodontitis,

\section{Introduction}

Periodontal diseases (gingivitis and periodontitis) are among the most widespread chronic diseases affecting populations worldwide [1]. Gingivitis is characterised by inflammation of the gums caused by plaque deposits, with possible bleeding when brushed or probed [2]. Gingivitis is limited to inflammation involving only the gingival soft tissues. Gingivitis has a high prevalence rate and affects $50 \%-90 \%$ of adults worldwide $[3,4]$

In its severest form, periodontitis, initiated by plaque biofilm, results in a loss of periodontal attachment to the root surface and adjacent alveolar bone, which 
ultimately leads to tooth loss $[5,6]$. Prevalence rates of periodontitis among people aged 35-44 years are 54\% in Taiwan and 47\% in the United States [7].

Periodontal disease is widely believed to be initiated by microbial interaction, which triggers a host response by setting off an inflammatory reaction [8]. Specific bacteria induce the release of cytokines thatincrease the number of polymorphonuclear leukocytes (PMNs) and their activity. Simultaneously, PMNs produce and increase the concentration of reactive oxygen species (ROS) resulting in oxidative damage to the periodontal tissue. These destructive mechanisms include damage toDNA, lipid peroxidation, and protein and enzyme oxidation $[9,10,11]$. ROS and oxidative stress might be major contributors to the pathogenesis of several chronic degenerative diseases [12,13].

The host can eliminate ROS and inhibit tissue destruction caused by inflammatory reactions; various defence mechanisms are derived from host saliva. An important defence mechanism is the antioxidant system; an important antioxidant is superoxide dismutase (SOD). SOD catalyses superoxide anions and defends the cells against the hazardous effects of ROS $[9,12]$.

There are controversial reports of the relationship between SOD and inflammation in the oral cavity. Periodontal disease has been observed with lower levels of serum SOD compared to healthy patients, and the difference was found to be significant [4]. Antioxidant concentrations have been observed to progressively decrease from healthy subjects to patients with gingivitis and periodontitis [80]. In contrast, Moore et al. [14] and Wei et al. [15] reported that SOD values were significantly higher in a chronic periodontitis group compared to a control group $[14,15]$. The purpose of this study was to determine the differences in SOD levels in saliva derived from gingivitis and periodontitis patients.

\section{Methods}

\subsection{Patient selection and clinical measurements}

The study's subjects were patients from the periodontal installation of the Dentistry Faculty, University of Sumatera Utara (USU), Medan, Indonesia. Informed consent was obtained from subjects willing to participate in the study. Ethical clearance for the study was received from the Health Research Ethical Committee, Medical Faculty, USU.

Forty-four patients were selected; 22 were gingivitis patients and 22 were periodontitis patients all within the age range of 20-60 years with more than 20 functional teeth. The subject's periodontal status was determined by measuring pocketprobing depths (PDs); clinical attachment levels (CALs); plaque index (PI) [16]; gingival index (GI) [17]; calculus index and papillary bleeding index (PBI) [17]. Gingivitis subjects were categorised by the following criteria: GI $>1$, pocket PDs $<4 \mathrm{~mm}$ and absence of clinical attachment loss and bone loss determined radiographically. Subjects with periodontitis were considered if at least two non-adjacent sites per quadrant with pocketPDs $>5 \mathrm{~mm}$ were present, clinical attachment loss, and radiographically determined bone loss. 
The exclusion criteria were medical conditions that could affect the results, subjects that had taken vitamins, anti-inflammatory drugs or antibiotics in the three months prior, pregnant women, lactating woman, smokers and alcoholics.

\subsection{Collection of samples}

Unstimulated whole saliva samples were collected using as pitting method. Seated patients were instructed to allow saliva to pool in the bottom of their mouth and drain into a collection tube. Saliva collection was performed for 5 minutes with a 1 minute interval to remove the saliva into a collection tube; up to $2 \mathrm{ml}$ of saliva was collected from each subject. The collected saliva was taken to the laboratory at the USU medical faculty in a cooling box. Collected saliva was stored in the freezer ($80^{\circ} \mathrm{C}$ ) if not checked immediately.

\subsection{Salivary SOD analysis}

Collected saliva was transferred from the collection tubes to Eppendorf tubes and centrifuged at $10000 \mathrm{rpm}$ for 60 seconds at $7^{\circ} \mathrm{C}$ before analysis. Salivary SOD levels were measured using an EnzyChrom ${ }^{\mathrm{TM}}$ Superoxide Dismutase Assay Kit (ESOD100) which included $20 \mathrm{~mL}$ assay buffer, $120 \mu \mathrm{L}$ SOD enzyme, $600 \mu$ Lxanthine, 20 mLdiluent, $120 \mu \mathrm{L}$ XO enzyme, and600 $\mu \mathrm{L}$ WST-1. Salivary SOD levels were measured using a spectrophotometer.

\subsection{Statistical analysis}

Using the Shapiro-Wilk test, the distribution of each data was tested for normality before analysis. An independent t-test was used to examine differences in salivary SOD levels of gingivitis and periodontitis patients.

\section{Results}

The study's demographic data included gender, age group, tooth brushing frequency, tooth brushing time and time of last visit to a dentist (Table 1).

Most of the subjects were women; the gingivitis group was comprised of subjects aged less than 30 years old, and the periodontitis group was predominantly aged $30-50$ years $(78.6 \%)$

All subjects brushed at least once daily; the predominant tooth brushing frequency for both groups was 2-3 times a day. The brushing routine, for both groups, was mostly done at bath time. In the gingivitis group, as many as 14 subjects $(60.9 \%)$ had visited a dentist in the last three months. In the periodontitis group, the number of subjects who had visited the dentist $1-3$ months ago $(56.3 \%)$ was equal to the number of subjects who had visited the dentist within the last three months $(39.1 \%)$.

The average score of plaque index, gingival index, calculus index, and papillary bleeding index were higher on the periodontitis group than gingivitis group (Table 2). 
Table 1. Demographic data of gingivitis patients and periodontitis patients

\begin{tabular}{|l|c|c|c|}
\hline \multicolumn{1}{|c|}{ Variable } & $\begin{array}{c}\text { Gingivitis } \\
\text { N(\%) }\end{array}$ & $\begin{array}{c}\text { Periodontitis } \\
\text { N(\%) }\end{array}$ & Total \\
\hline Gender: & $6(46.2)$ & $7(53.8)$ & 13 \\
a. Male & $16(51.6)$ & $15(48.4)$ & 31 \\
b. Female & $19(100)$ & & \\
\hline Aged: & $3(21.4)$ & $11(78.6)$ & 19 \\
a. <30 years old & $0(0)$ & $11(100)$ & 14 \\
b. 30-50 years old & & & \\
c. >50 years old & $1(33.3)$ & $2(66.7)$ & 3 \\
\hline Tooth-brushing Frequency: & $21(52.5)$ & $19(47.5)$ & 40 \\
a. 1 times/day & $0(0)$ & $1(100)$ & 1 \\
b. 2-3 times/day & & & \\
c. >3 times/day & $1(25)$ & $3(75)$ & 4 \\
\hline Tooth-brushing Time: & $20(54.1)$ & $17(45.9)$ & 37 \\
a. After breakfast and before bed & $1(33.3)$ & $2(66.7)$ & 3 \\
b. Every bath time & & & \\
d. Morning only or evening only & $1(20)$ & $4(80)$ & 5 \\
\hline Dental visit pattern: & $7(43.8)$ & $9(56.3)$ & 16 \\
a. 1-4 weeks ago & $14(60.9)$ & $9(39.1)$ & 23 \\
b. 1-3 months ago & &
\end{tabular}

Table 2. Clinical examination of the oral cavity of gingivitis and periodontitis patients

\begin{tabular}{|c|c|c|}
\hline Variable & $\begin{array}{c}\text { Gingivitis (n=22) } \\
\text { Mean } \pm \text { SD }\end{array}$ & $\begin{array}{c}\text { Periodontitis (n=22) } \\
\text { Mean } \pm \text { SD }\end{array}$ \\
\hline Plaque Index & $59.34 \pm 15.83$ & $64.08 \pm 13.34$ \\
\hline Gingival Index & $0.80 \pm 0.40$ & $1.005 \pm 0.52$ \\
\hline Calculus Index & $1.21 \pm 0.58$ & $1.61 \pm 0.44$ \\
\hline Papillary Bleeding Index & $50.77 \pm 16.75$ & $55.63 \pm 24.56$ \\
\hline
\end{tabular}

Table 3. Normality test of SOD levels in gingivitis and periodontitis group

\begin{tabular}{|c|c|c|}
\hline Group & $\mathbf{x} \pm$ SD & $\mathbf{p}$ \\
\hline Gingivitis $(\mathrm{n}=22)$ & $12.44 \pm 4.43$ & 0.377 \\
\hline Periodontitis $(\mathrm{n}=22)$ & $9.19 \pm 3.74$ & 0.228 \\
\hline
\end{tabular}

Shapiro-Wilk test; significant $p>0.05$

The normality test used in this research was the Shapiro-Wilk test. The p values of these two groups indicated that the data was normally distributed $(p>0.05)$ (Table 3$)$.

Table 4. The Difference of SOD levels in gingivitis and periodontitis group

\begin{tabular}{|c|c|c|}
\hline Group & $\mathbf{x} \pm$ SD & $\mathbf{p}(\mathbf{s i g})$ \\
\hline Gingivitis & $12.44 \pm 4.43$ & \multirow{2}{*}{0.012} \\
\hline Periodontitis & $9.19 \pm 3.74$ & \\
\hline
\end{tabular}

Independent T-test; significant $\mathrm{p}<0.05$ 
The difference in SOD levels between the groups was calculated using an independent $t$-test; the average SOD level for the gingivitis group was higher than for the periodontitis group. and the difference was statistically significant $(\mathrm{p}=0.012)$ (Table $4)$.

\section{Discussion}

In gingivitis and periodontitis patients, the host has evolved a highly sophisticated and complex antioxidant protection system to protect the periodontal tissue against ROS [18]. One of the antioxidants that can overcome ROS and inhibit oxidative stress is SOD; it is localised within human periodontal ligament and may represent a primary defence mechanism within gingival fibroblasts against release of excess superoxide [19].Several studies have examined the association of antioxidants with periodontal disease and the association of SOD with gingivitis and periodontitis disease. Thomas et al. [5] reported that SOD level were higher in healthy people compared to gingivitis and periodontitis patients. There findings were confirmedby Karim's [8] research that showed that the concentration of antioxidants in periodontitis patientswas lower compared to people with gingivitis and healthy people. Both studies were also confirmed by Novakovic's [20] research that showed a decrease in SOD activity in patients with chronic periodontitis compared with healthy individuals, and the decreased SOD activity was accompanied by reduced antioxidant concentration. Additionally, Kim's [12] research was also in agreement: higher SOD activity was found in healthy controls compared to patients with chronic periodontitis.

In contrast, Moore et al. [14] showed that there was no difference between the total number of salivary antioxidants in patients with chronic periodontitis andhealthy people. Moore et al.'s[14] research was supported by Wei's [15] study of SOD concentrations, total oxidative status and melano-di-aldehyde levels in people with periodontal disease. Wei's [15] research suggests that SOD is higher in individuals with periodontitis compared to healthy individuals.

The results of the current study showed a significant difference $(p<0.05)$ in salivary SOD levels between gingivitis patients and periodontitis patients; gingivitis patients had higher SOD levels compared to patients with periodontitis. The results of this study agree with Thomas [5], Karim [8], Novakovic [20] and Kim's [12] studies; SOD in patients with periodontal disease was lower than healthy people.This is due to an inflammatory relationship that occurs in periodontal disease with the antioxidant activity of the body. In unhealthy individuals, there is an increase in oxidative stress due to higher oxidant counts compared to some antioxidants, whereas in healthy individuals, there is a balance between oxidants and antioxidant counts [21]. Decreased antioxidants and increased oxidative stress leads to increased periodontal tissue damage in gingivitis and periodontitis. The decline in antioxidants and the increasing state of oxidative stress with continued inflammation, if not promptly treated, will exacerbate periodontal tissue damage and result in tooth loss [8].

The results of the current study also showed a difference in the severity of inflammation occurring with gingivitis and periodontitis; periodontitis inflammation is more severe than gingivitis inflammation. This was confirmed by the results of 
the oral examinations performed on both groups. In the periodontitis group, there were increased PDs,an average pocket depth of6.22 $\mathrm{mm}$ and attachment loss. Higher inflammatory activity in periodontitis compared to gingivitis results in lower antioxidants associated with periodontitis compared to gingivitis; one of the declining antioxidants is SOD. There was a decrease in SOD with a probing depth of more than six mm [8].This couldbe one of the causes of the higher SOD levels in the gingivitis group compared to the periodontitis group.

However, the results of the current study contradict Moore et al.'s [14] where no difference was found between the total number of antioxidants in the saliva of people with periodontitis and healthy people. The results of the current study are also in contrast to Wei et al. [15] that showed higher SOD counts in patients with chronic periodontitis compared to healthy patients.

These studies' differing results may have occurred because of several factors, including different inclusion and exclusion criteria, sample differences and research methods. In Wei et al.'s [15] research, the subjects were patients with chronic periodontitis compared with healthy controls. In the current study, both groups of subjects were patients with periodontal disease. Moore et al.'s [14] research included only seven patients with periodontal disease who were not balanced with the number of healthy samples. In the current study, the two sample groups were balanced; each consisted of 22 individuals.

SOD is one of the antioxidants that plays an important role in the development of periodontal tissue inflammation. SOD functions by catalysing superoxide that protects body cells and prevents the inflammation process caused by free radicals. SOD can be produced in the body by itself, but the antioxidant cannot work optimally if not induced by secondary antioxidant intake derived from food. The higher intake of antioxidants derived from food improves maintain of antioxidants in the body [22].

\section{Conclusions}

This research concluded that there was a difference between salivary SOD levels in gingivitis and periodontitis patients. Patients with gingivitis had significantly higher salivary SOD levels compared to patients with periodontitis.

\section{Acknowledgements}

The authors would like to extend their appreciation to those who helped in this research including all staff of Installation of Periodontia, Faculty of Dentistry USU and all staff of Laboratory at Medical Faculty of USU.

\section{References}

1. Thomson WM, Sheiham A, Spencer AJ. Sociobehavioral aspects of periodontal disease. Periodontology 2000. 2012;60(1):54-63.

2. Sculley DV, Langley-Evans SC. Salivary antioxidants and periodontal disease status. Proceedings of the Nutrition Society. 2002;61(1):137-43.

3. Cobb CM. Microbes, inflammation, scaling and root planing, and the periodontal condition. Ame Dent Hyg Assoc. 2008;82(suppl 2):4-9. 
4. Fiorellini JP, Stathopolou PG. Clinical Features of Gingivitis. In: Carranza, Forrest, Jepsen, Klokkevold, Newman, Preshaw, Takei, Teughels, eds. J Clin Periodontol. 12th ed. St. Louis, Missouri: Elsevier Saunder; 2015:224.

5. Biju T, Shabeer MM, Amitha R, Rajendra BP, Suchetha K. Comparative evaluation of serum superoxide dismutase and glutathione levels in periodontally diseased patients: an interventional study. Indian J Dent Res. 2014;25(5):613.

6. Hinrichs JE, Kotsakis G. Classification of Disease and Condition Affecting the Periodontium. In: Carranza, Forrest, Jepsen, Klokkevold, Newman, Preshaw, Takei, Teughels. J Clin Periodontol. 12th ed. St. Louis, Missouri: Elsevier Saunder; 2015:45-55.

7. Yang PS, Huang WC, Chen SY, Chen CH, Lee CY, Lin CT, Huang YK. Scaling-stimulated salivary antioxidant changes and oral-health behavior in an evaluation of periodontal treatment outcomes. Scientific World J. 2014;2014.

8. Karim S, Pratibha PK, Kamath S, Bhat GS, Kamath U, Dutta B, Sharma N, Archana B, Bhat KM, Guddattu V. Superoxide dismutase enzyme and thiol antioxidants in gingival crevicular fluid and saliva. Dent Res J. 2012;9(3):266.

9. Ahmadi MF, et al. Oxidative Stress and Antioxidants. DJH 2011;3(1):1-11.

10. Bhusari BM, Mahajan R, Rajbhoj S, Shah P. Reactive Oxygen Species \& Its Role in Periodontal Disease. J Dental Med Sci. 2014; 13:52-9.

11. Guentsch A, Preshaw PM, Bremer-Streck S, Klinger G, Glockmann E, Sigusch BW. Lipid peroxidation and antioxidant activity in saliva of periodontitis patients: effect of smoking and periodontal treatment. Clinical oral investigations. 2008;12(4):345.

12. Kim SC, Kim OS, Kim OJ, Kim YJ, Chung HJ. Antioxidant profile of whole saliva after scaling and root planing in periodontal disease. J Periodont Impl Sci. $2010 ; 40(4): 164-71$.

13. Pendyala G, Thomas B, Kumari S. The challenge of antioxidants to free radicals in periodontitis. J Indian Soc Periodontol. 2008;12(3):79.

14. Shirzaiy M, Ansari SM, Dehghan JH, Ghaeni SH. Total anti-oxidant capacity of saliva in chronic periodontitis patients before and after periodontal treatment. J Nepal Health Research Council. 2015 Apr 20.

15. Wei D, Zhang XL, Wang YZ, Yang CX, Chen G. Lipid peroxidation levels, total oxidant status and superoxide dismutase in serum, saliva and gingival crevicular fluid in chronic periodontitis patients before and after periodontal therapy. Australian Dent J. 2010;55(1):70-8.

16. Moslehzadeh K. Oral Health Database. Available at : " http : // www.mah.se / CAPP / Methods-and-Indices / Oral-Hygiene-Indices / Silness-Loe-Index / " . Accessed February $18,2017$.

17. Bessa Rebelo MA, Correa de Queiroz. Gingival Indices: State of Art, Gingival DiseasesTheir Aetiology, Prevention and Treatment. Available at: "http://www.intechopen.com/books/gingival-diseases-their-aetiology-prevention-andtreatment/gingival-indices-state-of-art”. Accessed February 18, 2017.

18. Carnelio S. Definite, Probable or Dubious : Antioxidants Trilogy in Clinical Dentistry. Br Dent J2008; 204(1):29-31.

19. Sree SL, Mythilli R. Antioxidants in Periodontal Diseases: A Review. Indian J Multidiciplinary Dent 2011;1(3):140-6.

20. Novakovic N, Cakic S, Todorovic T, Ralcevic BA, Dozic I, Petrovic V et al. Antioxidant Status of Saliva Before and After Non-Surgical Periodontal Treatment. J Periodontal Res 2014;49:129-36.

21.Azizi A, Sariati F, Parchakani A, Alirezaei S. Evaluation of Whole Saliva Antioxidant Capacity in Patients with Periodontal Disease. Open J Stomatol2014;4:228-31.

22. Winarsi H. Natural Antioxidants and Free Radicals: Potential and Its Application in Health. Yogyakarta: Kanisius, 2007:20-3. 